\title{
A CHARACTERIZATION OF ALL LOGLINEAR INEQUALITIES FOR THREE QUERMASSINTEGRALS OF CONVEX BODIES
}

\author{
PETER GRITZMANN
}

(Communicated by William J. Davis)

\begin{abstract}
We give a complete characterization of all inequalities of the type $W_{i}^{\alpha}(K) W_{j}^{\beta}(K) W_{k}^{\gamma}(K) \geq c$, where $K$ is an arbitrary convex body of Euclidean $d$-space, $W_{l}(K), l=i, j, k$, denotes the $l$ th quermassintegral of $K$ and $\alpha, \beta, \gamma$ and $c$ are arbitrary reals. A special case of such inequalities is the classical isoperimetric inequality for the volume and surface area of convex bodies. It turns out that all nontrivial inequalities of this type can be generated by means of $d-1$ basic inequalities, the Fenchel-Alexandrov inequalities.
\end{abstract}

1. Introduction and results. The classical isoperimetric inequality

$$
d^{d} \omega_{d} V^{d-1}(K) \leq S^{d}(K)
$$

for the volume $V(K)$ and the surface area $S(K)$ of arbitrary convex bodies in Euclidean $d$-space $E^{d}$ is one of the most famous results in geometry. Here $\omega_{d}$ denotes the volume of the unit ball $B^{d}$ and the constant $d^{d} \omega_{d}$ is related to the fact that the inequality holds with equality if and only if $K$ is a ball. Meanwhile there is an extensive literature on generalizations, extensions and applications of this formula and we refer to $[\mathbf{4}, \mathbf{5}, \mathbf{7}$ and 19] for respective surveys.

In the present paper we give a characterization of all loglinear inequalities for three quermassintegrals.

Let $\mathscr{K}^{d}$ denote the set of convex bodies in Euclidean $d$-space $E^{d}$, i.e. the set of all compact, convex subsets of $E^{d}$ with nonempty interior. Furthermore, for $i=0, \ldots, d$ let $W_{i}$ denote the $i$ th quermassintegral. In particular, $W_{0}$ is the volume $V, d W_{1}$ is the surface area $S, d W_{2}$ is the integral $M$ of mean curvature, $\left(2 / \omega_{d}\right) W_{d-1}$ is the mean width $B$ and $W_{d}$ is equal to the volume $\omega_{d}$ of the unit ball $B^{d}$ (compare e.g. [15]).

Each of the quermassintegrals is a continuous, additive, monotone and positive functional on $\mathscr{K}^{d}$ which is invariant under arbitrary rigid motions and homogeneous of degree $d-i$.

The importance of the quermassintegrals is visible in Hadwiger's famous functional theorem $[11,12,13,14]$, see also $[15$, pp. 221-225] which states that every

Received by the editors June 18, 1987 and, in revised form, November 24, 1987.

1980 Mathematics Subject Classification (1985 Revision). Primary 52A40.

Key words and phrases. Isoperimetric inequality, quermassintegrals, convex bodies, FenchelAlexandrov inequalities.

Research was supported by the Alexander-von-Humboldt foundation and by the Institute for Mathematics and its Applications with funds provided by the National Science Foundation.

This paper was written while the author was visiting the University of Washington, Department of Mathematics, Seattle, Washington. 
continuous and additive functional on $\mathscr{K}^{d}$ which is invariant under rigid motions is a linear combination of the $d+1$ quermassintegrals.

Let us point out that these quermassintegrals are not dimensionally invariant, but using the renormalization

$$
\omega_{d-i} V_{i}=\left(\begin{array}{l}
d \\
i
\end{array}\right) W_{d-i}
$$

introduced in [18] we get the so-called intrinsic volumes $V_{0}, \ldots, V_{d}$ which have, of course, all properties of the quermassintegrals mentioned above but are, in addition, dimensionally invariant. (Furthermore, there is a nice interpretation of the $V_{i}$ for polytopes [18].)

One of the most challenging tasks concerning such functionals on $\mathscr{K}^{d}$ is the problem to find all inequalities which relate different quermassintegrals. In the present paper we are mainly concerned with inequalities of the following type

$$
W_{i}^{\alpha}(K) W_{j}^{\beta}(K) W_{k}^{\gamma}(K) \geq c
$$

where $K \in \mathscr{K}^{d}$ and

(4) $0 \leq i<j<k \leq d-1, \quad \alpha, \beta, \gamma, c \in \mathbf{R}, \quad \alpha^{2}+\beta^{2}+\gamma^{2} \neq 0 \quad$ and $\quad c>0$.

This includes, of course, inequalities of the form

$$
W_{i}^{\alpha}(K) W_{j}^{\beta}(K) W_{k}^{\gamma}(K) \leq c,
$$

since we allow negative exponents. So, (3) describes the general loglinear inequality for three different quermassintegrals. Obviously, (3) holds for $c \leq 0$, but all such inequalities are trivial. Thus, since $W_{d}$ is a constant the restriction (4) only excludes the most trivial cases.

We give the following characterization of all such inequalities.

THEOREM 1. Let $i, j, k, \alpha, \beta, \gamma$ satisfy (4). Then there is an inequality of type (3) if and only if

(5) $\quad \alpha \leq 0, \quad \alpha+\beta+\gamma \geq 0 \quad$ and $\quad \alpha(d-i)+\beta(d-j)+\gamma(d-k)=0$.

Furthermore, in all cases the constant $c$ can be chosen such that equality holds for the ball.

Actually, all nontrivial inequalities of this type are combinations of two families of inequalities.

$$
\begin{aligned}
\omega_{d} W_{i}^{d-i-1}(K) & \leq W_{i+1}^{d-i}(K) & & \text { for } i=0, \ldots, d-2 \\
W_{i}^{k-j}(K) W_{k}^{j-i}(K) & \leq W_{j}^{k-i}(K) & & \text { for } 0 \leq i<j<k \leq d-1 .
\end{aligned}
$$

Let us point out that the classical isoperimetric inequality (1) is the special case $i=0$ in (6).

These inequalities, in turn, can be generated by just $d-1$ basic inequalities, the Fenchel-Alexandrov inequalities $[\mathbf{1}, \mathbf{2}, \mathbf{3}, \mathbf{9}, \mathbf{1 0}]$

$$
W_{i-1}(K) W_{i+1}(K) \leq W_{i}^{2}(K) \text { for } i=1, \ldots, d-1
$$

for quermassintegrals of arbitrary convex bodies $K$. 
THEOREM 2. All inequalities of the form (3) subject to (5) are generated by the $d-1$ Fenchel-Alexandrov inequalities (8) and none of the inequalities (8) can be generated by the remaining $d-2$.

In this sense, the Fenchel-Alexandrov inequalities (8) form a basis of all loglinear inequalities for three quermassintegrals.

Let us point out that since

$$
W_{l}\left(B^{d}\right)=\omega_{d} \quad \text { for } l=0, \ldots, d
$$

equality holds in (8) in case of the ball. Furthermore, equality holds for $(d-i-1)$ tangential bodies of a ball (see $[\mathbf{7}, \mathbf{2 0}]$ ), thus, if $i \leq d-2$ particularly for the convex hull of $B^{d}$ and a line segment centered at the origin. For a survey on the FenchelAlexandrov inequalities and, particularly, on the state of the art of the discussion of the equality case (which has not been settled, yet, completely) and for references see $[20]$.

We will show the necessity of the conditions (5) of Theorem 1 in the next section and in $\S 3$ we show that all such inequalities are generated by (8), thus proving Theorems 1 and 2.

Using these results we can get characterizations of inequalities for some other functionals which are associated with convex bodies. We demonstrate this in the case of the diameter. Particularly, we obtain a short proof of an isodiametric inequality involving the volume and the surface area given in [8] and its generalization to arbitrary quermassintegrals.

The notation used below is quite standard. For concepts, notation and abbreviations not explained below we refer to $[\mathbf{7}, \mathbf{1 5}$ or 17].

2. The necessity of the condition. In this section we show that the conditions (5) are, indeed, necessary for the existence of an inequality of type (3), (4) for arbitrary convex bodies $K$.

Since we are not concerned with special values of the constant $c$, throughout this section the occurrence of $c$ is to be understood as the statement that there exists a constant $c$ such that the current inequality holds for all convex bodies.

Since both sides in (3) are positive we may consider inequality (3) in the "linear form"

(9) $\alpha L_{i}(K)+\beta L_{j}(K)+\gamma L_{k}(K) \geq c \quad$ where $L_{l}(K)=\log \left[W_{l}(K)\right]$ for $l=i, j, k$.

Clearly, we now have to allow $c$ to be an arbitrary real. Since the $l$ th quermassintegral is homogeneous of degree $d-l$ the inequality (9) implies that for all positive real $r$

$\alpha\left(L_{i}(K)+(d-i) \log r\right)+\beta\left(L_{j}(K)+(d-j) \log r\right)+\gamma\left(L_{k}(K)+(d-k) \log r\right) \geq c$, which means, of course, that

$$
\alpha(d-i)+\beta(d-j)+\gamma(d-k)=0 .
$$

Observing that for every $l \leq d-1$ there is a sequence $\left(K_{n}\right)_{n \in \mathbf{N}}$ of convex bodies such that $W_{l}\left(K_{n}\right)$ tends to 0 as $n \rightarrow \infty$ but the sequence $\left(W_{m}\left(K_{n}\right)\right)_{n \in \mathrm{N}}$ is bounded away from zero whenever $l<m$ it follows that the first nonzero among the coefficients $\alpha, \beta, \gamma$ must be negative. In any case,

$$
\alpha \leq 0 \text {. }
$$


Now, suppose $Z$ is a right cylinder of height $h$ over the $(d-1)$-dimensional unit ball. Then we have

$$
W_{l}(Z)=\frac{\omega_{d-1}}{d}\left(\frac{l \omega_{l}}{\omega_{l-1}}+(d-l) h\right)
$$

(compare [15, p. 219]). Thus, when applied to $Z$ inequality (9) becomes

$$
\begin{aligned}
\alpha \log \left(\frac{i \omega_{i}}{\omega_{i-1}}+(d-i) h\right) & +\beta \log \left(\frac{j \omega_{j}}{\omega_{j-1}}+(d-j) h\right) \\
& +\gamma \log \left(\frac{k \omega_{k}}{\omega_{k-1}}+(d-k) h\right) \geq c
\end{aligned}
$$

and for $h \rightarrow \infty$ this inequality can only hold if

$$
\alpha+\beta+\gamma \geq 0 \text {. }
$$

Obviously, conditions (10), (11) and (12) together give condition (5).

Let us, finally, point out that for the proof of the necessity of the condition we did not need all specific properties of the quermassintegrals. Accordingly, the result holds for a larger class of families of functionals.

3. Generating loglinear inequalities. Now we show that for all $i, j, k, \alpha, \beta, \gamma$ subject to (4) and (5) there exist inequalities of type (3). Again, at first, we do not deal with specific constants. Optimal constants will be given at the very end of this section.

In order to prove Theorem 1 we first reduce all cases to six series of possible inequalities, then we show that all six can be generated by means of the two series (6) and (7) and finally we show these inequalities can be generated by the FenchelAlexandrov inequalities (8).

For $\alpha=0$ the equality condition in (5) implies that $\beta \gamma<0$. Suppose $\gamma<0$ and $\beta>0$. Then by the second inequality (5) $\beta \geq|\gamma|$. Thus, since $d-j>d-k$ the equality condition in (5) is not satisfied. This contradiction shows that the only possibility for $\alpha=0$ is

$$
\alpha=0, \quad \beta<0, \quad \gamma>0
$$

Assume, now, that $\alpha$ is negative. Since by (5) not all three exponents can be nonpositive only the following cases are possible.

$$
\begin{array}{lll}
\alpha<0, & \beta=0, & \gamma>0 \\
\alpha<0, & \beta<0, & \gamma>0 \\
\alpha<0, & \beta>0, & \gamma=0 \\
\alpha<0, & \beta>0, & \gamma<0 \\
\alpha<0, & \beta>0, & \gamma>0 .
\end{array}
$$

For the second part it seems to be more appropriate to deal with inequalities of type (9) rather than with those of type (3) (although we have to allow $c$ to be an arbitrary real). For convenience we, moreover, suppress the argument $K$. Since (9) is positively homogeneous all possible inequalities in the cases (13), (14) and (16) reduce to the following two-parameter series of inequalities:

$$
-(d-m) L_{l}+(d-l) L_{m} \geq c \text { for } l=0, \ldots, d-2, m=l+1, \ldots, d-1
$$


which, in turn, can be generated by the one-parameter series

$$
-(d-i-1) L_{i}+(d-i) L_{i+1} \geq c \text { for } i=0, \ldots, d-2 .
$$

Except for the nonspecified constant this series is equivalent to (6).

Now, suppose our coefficients satisfy (5) and (15). Set

$$
\gamma_{1}=|\alpha| \frac{d-i}{d-k} \quad \text { and } \quad \gamma_{2}=|\beta| \frac{d-j}{d-k}
$$

the triples $\left(\alpha, 0, \gamma_{1}\right)$ and $\left(0, \beta, \gamma_{2}\right)$ of parameters satisfy (5), respectively, and we have $\gamma_{1}+\gamma_{2}=\gamma$. So, if there exist all inequalities of type (19), and thus there exist all inequalities with coefficients satisfying (13) or (14) then we also have inequalities with coefficients subject to (15), in general. Equally well, the case (18) can be reduced to (19) but not the case (17).

Now, we deal with the case (17). Assume, first,

$$
\alpha+\beta+\gamma>0 \text {. }
$$

Setting

$$
\begin{gathered}
\alpha_{1}=\alpha \frac{d-i}{j-i}+\beta \frac{d-j}{j-i}+\gamma \frac{d-j}{j-i} \\
\beta_{1}=-\gamma-\alpha_{1}, \quad \beta_{2}=\beta-\beta_{1}, \quad \alpha_{2}=-\beta_{2} \frac{d-j}{d-i}
\end{gathered}
$$

we see that the triples $\left(\alpha_{1}, \beta_{1}, \gamma\right)$ and $\left(\alpha_{2}, \beta_{2}, 0\right)$ of coefficients fit into the cases (17), (16), respectively, that (5) holds, and that we have

$$
\alpha_{1}+\alpha_{2}=\alpha, \quad \beta_{1}+\beta_{2}=\beta \quad \text { and } \alpha_{1}+\beta_{1}+\gamma=0 \text {. }
$$

So, assuming that the general inequality in case (19) holds and, thus, (16) we may further reduce the case (17) to $\alpha+\beta+\gamma=0$. Thus we have

$$
\alpha<0, \quad \beta>0, \quad \gamma<0, \quad \alpha+\beta+\gamma=0 \quad \text { and } \alpha i+\beta j+\gamma k=0 .
$$

Solving the equalities in $(20)$ we get

$$
\frac{\alpha}{\gamma}=\frac{k-j}{j-i} \quad \text { and } \quad \frac{\beta}{\gamma}=\frac{i-k}{j-i} \text {. }
$$

Since (7) is positive homogeneous (21) shows that (17) can be reduced to (7).

In conclusion, all cases (13)-(18) can be reduced to (6) and (7).

To show that (6) and (7) hold and, simultaneously, to prove Theorem 2 we have to generate all such inequalities by means of the Fenchel-Alexandrov inequalities (8).

Observe, first, that it suffices to deduce all inequalities of the form

$$
W_{i}^{j-k}(K) W_{j}^{k-i}(K) W_{k}^{i-j}(K) \geq 1, \quad 0 \leq i<j<k \leq d .
$$

By allowing the case $k=d$ we include the type (6).

Let us, again, switch to the respective logarithmic inequality of type (9) and let us identify the inequalities with a $(d+1)$-dimensional vector

$$
z^{i, j, k}=\left(\varsigma_{0}^{i, j, k}, \ldots, \varsigma_{d}^{i, j, k}\right)^{T} \quad \text { defined by } \varsigma_{l}^{i, j, k}= \begin{cases}j-k & \text { for } l=i \\ k-i & \text { for } l=j \\ i-j & \text { for } l=k \\ 0 & \text { else. }\end{cases}
$$


To show that the inequalities (8) generate (22) we have to show that the following system of linear inequalities is always feasible:

$$
\sum_{n=1}^{d-1} z^{n-1, n, n+1} \xi_{n}=z^{i, j, k}, \quad \xi_{1}, \ldots, \xi_{d-1} \geq 0 .
$$

Denote the column vector of all variables by $x$ and the coefficient matrix by $A$; the system becomes in matrix notation

$$
A x=z^{i, j, k}, \quad x \geq 0
$$

and we have

$$
A=\left(\begin{array}{rrrrrr}
-1 & 0 & 0 & 0 & \ldots & 0 \\
2 & -1 & 0 & 0 & \ldots & 0 \\
-1 & 2 & -1 & 0 & \ldots & 0 \\
0 & -1 & 2 & -1 & \ldots & 0 \\
\vdots & \vdots & \vdots & \vdots & \ddots & \vdots \\
0 & 0 & 0 & 0 & \ldots & -1 \\
0 & 0 & 0 & 0 & \ldots & 2 \\
0 & 0 & 0 & 0 & \ldots & -1
\end{array}\right)
$$

Observe that the system $A x=z^{i, j, k}$ of linear equations has a unique solution since the columns of $A$ are linearly independent and $z^{i, j, k}$ is orthogonal to the left nullspace of $A$. Now, it is easy to see that $-B$ is a left inverse of $A$, where

$$
B=\left(\begin{array}{cccccccc}
1 & 0 & 0 & 0 & \cdots & 0 & 0 & 0 \\
2 & 1 & 0 & 0 & \cdots & 0 & 0 & 0 \\
3 & 2 & 1 & 0 & \cdots & 0 & 0 & 0 \\
4 & 3 & 2 & 1 & \cdots & 0 & 0 & 0 \\
\vdots & \vdots & \vdots & \vdots & \ddots & \vdots & \vdots & \vdots \\
d-1 & d-2 & d-3 & d-4 & \cdots & 1 & 0 & 0
\end{array}\right)
$$

Let $e_{l}$ denote the $(l+1)$ th cartesian unit vector of $E^{d+1}$. Then

$$
x=-B A x=-B z^{i, j, k}=(k-j) B e_{i}-(k-i) B e_{j}+(j-i) B e_{k} .
$$

Now, let $n \in\{1, \ldots, d-1\}$. Then $\xi_{n} \geq 0$ for $n \leq j$. For $j+1 \leq n \leq k$ we calculate

$$
\xi_{n}=(k-j)(n-i)-(k-i)(n-j)=(k-n)(j-i) \geq 0 .
$$

Furthermore, for $k+1 \leq n \leq d-1$ we have $\xi_{n}=0$. Thus, (23) is feasible.

It follows that we can, indeed, generate all the inequalities in question by means of (8), which proves Theorem 2 and Theorem 1 except for the statement concerning the choice of the constant $c$.

But since (8) holds with equality for $B^{d}$, and since equality is preserved by the operations performed to reduce all possible cases to (8) we can choose the constant $c$ in (3) so as to achieve equality. This completes the proof of Theorem 1.

4. On isodiametric inequalities. In this final section we apply our theorems in order to characterize all loglinear inequalities for the diameter $D$ and two quermassintegrals of convex bodies. Well-known examples of such inequalities are Bieberbach's formula [6]

$$
V(K) \leq \omega_{d}\left(\frac{D(K)}{2}\right)^{d}
$$


and Kubota's inequality [16]

$$
S(K) \leq d \omega_{d}\left(\frac{D(K)}{2}\right)^{d-1}
$$

Corollary. Let $0 \leq i<j \leq d-2$, let $\alpha, \beta, \gamma, c \in \mathbf{R}, \alpha^{2}+\beta^{2}+\gamma^{2} \neq 0, c>0$. Then there is an inequality of the type

$$
W_{i}^{\alpha}(K) W_{j}^{\beta}(K) D^{\gamma}(K) \geq c
$$

for all convex bodies $K$ of $\mathscr{K}^{d}$ if and only if

$$
\alpha \leq 0, \quad \alpha+\beta+\gamma \geq 0 \quad \text { and } \quad \alpha(d-i)+\beta(d-j)+\gamma=0 .
$$

PROOF. To prove this result, all we have to do is to replace $W_{d-1}(K)$ by $D(K)$. That this can be done is due to the following inequalities.

$$
\frac{\omega_{d-1}}{d} D(K)<W_{d-1}(K) \leq \frac{\omega_{d}}{2} D(K) \text {. }
$$

The strict inequality follows from the monotonicity of $W_{d-1}$ (or, more easily, from the monotonicity of $V_{1}$; see (2) and the second inequality simply uses the definition of the mean width.

Let us point out that the strict inequality in (25) would hold with equality for line segments (but we have restricted $\mathscr{K}^{d}$ to bodies with interior points) while the second inequality holds with equality only for bodies of constant width. So, unlike Theorem 1, in the Corollary the constants $c$ cannot always be chosen to give equality for at least one convex body. However, using the fact that (8) holds with equality for $(d-i-1)$-tangential bodies of $B^{d}$ and the continuity of the quermassintegrals and the diameter, or using directly the formula

$$
W_{l}(C)=\frac{W_{d-1}}{d}\left(h+\int_{\pi / 2-\arctan (2 / h)}^{\pi / 2+\arctan (2 / h)} \frac{1-\sin ^{l} \tau}{\cos ^{2} \tau} d \tau\right)
$$

for the $l$ th quermassintegral of a double cone over $B^{d-1}$ of heights $h$ (see $[\mathbf{1 5}, \mathrm{pp}$. 217-220]), we see that in most cases the (multiplicative) constant which we get from (6) or (7) and (25) is best possible since in the respective inequalities either equality occurs for balls or they are at least asymptotically optimal for double cones $C$ over the $(d-1)$-dimensional unit ball with heights $h$ tending to $\infty$.

Let us finally remark that the case $i=0, j=1$ in (24), i.e. the inequality

$$
S^{d-1}(K)>\omega_{d-1} D(K)[d V(K)]^{d-2}
$$

was first proved in [8] using a completely different and direct approach which was based on symmetrization arguments and an inequality for integral means of concave functionals.

\section{REFERENCES}

1. A. D. Alexandrov, Neue Ungleichungen für die Mischvolumen konvexer Körper, C. R. (Doklady) Acad. Sci. URSS 14 (1937), 155-157.

2. __ Zur Theorie der gemischten Volumina von konvexen Körpern. II. Neue Ungleichungen zwischen den gemischten Volumina und ihre Anwendungen, Rec. Math. N.S.=Mat. Sb. 2 (1937), 1205-1238. (Russian) 
3. __ Zur Theorie der gemischten Volumina von konvexen Körpen. IV. Gemischte Diskriminanten und gemischte Volumina, N.S. Rec. Math.=Mat. Sb. 3 (1938), 227-251. (Russian)

4. C. Bandle, Isoperimetric inequalities and applications, Pitman, London, 1980.

5. __ Isoperimetric inequalities, Convexity and Its Applications (P. M. Gruber and J. M. Wills, eds.), Birkhäuser, Basel, 1983, pp. 30-48.

6. L. Bieberbach, Über eine Extremaleigenschaft des Kreises, Jber. DMV 24 (1915), 247-250.

7. T. Bonnesen and W. Fenchel, Theorie der konvexen Körper, Springer-Verlag, Berlin, 1934.

8. P. Gritzmann, J. M. Wills, and D. Wrase. A new isoperimetric inequality, J. Reine Angew. Math. 379 (1987), 22-30.

9. W. Fenchel, Inégalités quadratiques entre les volumes mixtes des corps convexes, C. R. Acad. Sci. (Paris) 203 (1936), 647-650.

10. ___ Généralisation du théorème de Brunn et Minkowski concernant les corps convexes, C. R. Acad. Sci. (Paris) 203 (1936), 764-766.

11. H. Hadwiger, Einige Anwendungen eines Funktionalsatzes für konvexe Körper in der räumlichen Integralgeometrie, Monatsh. Math. 54 (1950), 345-353.

12. (1951), 69-76.

13. __ Additive Funktionale k-dimensionaler Eichkörper I, Arch. Math. 3 (1952), 470-478.

14. __ Additive Funktionale k-dimensionaler Eichkörper II, Arch. Math. 4 (1953), 374-379.

15. __ Vorlesungen über Inhalt, Oberfläche und Isoperimetrie, Springer-Verlag, Berlin, 1957.

16. T. Kubota, Über konvexgeschlossene Mannigfaltigkeiten im $n$-dimensionalen Raume, Sci. Rep. Tôhoku Univ. 14 (1925), 85-99.

17. K. Leichtweiss, Konvexe Mengen, VEB Deutscher Verlag der Wissenschaften, Berlin, 1980.

18. P. McMullen, Nonlinear angle-sum relations for polyhedral cones and polytopes, Math. Proc. Cambridge Philos. Soc. 78 (1975), 247-261.

19. R. Ossermann, The isoperimetric inequality, Bull. Amer. Math. Soc. 84 (1987), 1182-1238.

20. R. Schneider, On the Fenchel-Aleksandrov inequalities, Discrete Geometry and Convexity (J. E. Goodman, E. Lutwak, J. Maikevitch and R. Pollack, eds.), Ann. N. Y. Acad. Sci., New York, 1985, pp. 132-141.

Department of Mathematics, University of Trier, D-5500 Trier, Federal REPUBLIC OF GERMANY 